\title{
Femoral Shaft Fractures in Children: A Comparative Study in Treatment with Spica and Tens
}

\author{
${ }^{1}$ Dr.R.Chandra Sekhar, ${ }^{2}$ Dr. J.Satya Prasad M.S., M.Ch \\ ${ }^{I}$.Postgraduate in Orthopaedics, KIMS ,Amalapuram, A.P. \\ 2. Professor \& HOD of Orthopaedics, KIMS, Amalapuram, A.P.
}

\section{Introduction}

Femoral shaft fractures in children are common and frequently lead to hospitalization and require anaesthesia for performing an orthopaedic procedure. Fractures of femur in children occur most frequently in the middle third of the shaft. According to Hinton et al, the annual rate of femoral shaft fractures in children is 19.5 per $1,00,000^{(1)}$. Femoral shaft fractures treatment goals in children are achieving bone union with length, alignment and limb's function restoration, without losing movements of adjacent joints. Femoral shaft fracture is an incapacitating pediatric injury. ${ }^{(2)}$ Femoral shaft fractures, including subtrochanteric and supracondylar fractures, represent approximately $1.6 \%$ of all body injuries in children The annual rate of femur shaft fractures in children was 1 per $5000^{(3)}$. However, incidence appears to show minor variations in its geographical distribution. Orthopaedic surgeons have long maintained that all children who have sustained a diaphyseal fracture of femur recover with conservative treatment, given the excellent remodeling ability of immature bone in children. But time and experience of many surgeons have shown that diaphyseal femur fractures in children do not always recover completely with conservative treatmen ${ }^{4)}$. Angulations, shortenings and malrotations are not always corrected by conservative treatment ${ }^{(5)}$.These fractures are commonly treated by conservative approach, with reduction and early immobilization with plaster cast or preceded by skin or skeletal traction. This procedure is not exempted from complications, with reduction loss and the shortening of the affected limb.

Keywords-: Children, Femoral-shaft fracture, Spica cast, Titanium elastic nailing

Aims And Objectives:- . To study the functional outcome following the use of spica and TENS intramedullary flexible nails for treatment of femoral shaft fracture in paediatric age group from 1-12 years.. To study the time taken for union in each method of the above mentioned fractures. To study the complications of Spica and flexible intramedullary nailing for femoral shaft fracture

\section{Materials And Methods}

This is a retrospective study approved by the Committee on Ethics in Research of this Institution. Of the 123 children with ages ranging from 1 to 12 years with femoral shaft fractures during the period of 2014 to 2016 were included. Patients for whom insufficient data was available on medical files and those who presented no appropriate X-ray documentation for analysis were excluded from this study. Data gathered was name, registration, accident date, age, gender, side, location, exposure, mechanism of injury, related injuries, fracture trait, kind of treatment, complications, time for union, and clinical and X-ray review after union. We divided the children into three main groups. In Group I, constituted of children in the age group below 2 years old, the treatment of choice is reduction and immediate plastered cast; in Group II, represented by children between 2 years and 9 year, traction followed by plastered cast is usually employed, and; in Group III, comprised of children above the age of 10-12 years, surgical treatment is indicated

Procedure:-. Place the patient on an orthopaedic table, and reduce the fracture partially by traction guided by fluoroscopy.Entire segment of the involved limb on the injured side was thoroughly scrubbed with savlon and then painted with betadine and draped. A skin incision approximately $3 \mathrm{cms}$ long was made at the nail insertion site. Subcutaneous tissues are cleared with artery forceps. Periosteum is stripped with periosteal elevator and bone is exposed. An oval window of size $1 \mathrm{~cm}$ was made in the bone with a curved awl with the long axis in the vertical direction.

Use a 4- to $4.5-\mathrm{mm}$ drill bit with a tissue protector to make a hole in the cortex on the medial and lateral distal femoral metaphysis at a point approximately $3 \mathrm{~cm}$ proximal to the physis. Prepare the nails preoperatively by angling them at 45 degrees about $2 \mathrm{cms}$ from oneend to facilitate penetration of medullary canal, and bend them into an even curve over their entire length .

With the help of aT-handle and by rotation movements of the wrist, introduce the nails through a longitudinal drill hole. Use two nails one lateral and one medial, to stabilize the fracture. Carefully push both up the medullary canal to the already reduced fracture site. After touching the opposite internal cortex, the nails 
bend themselves in the direction of the long bone's axis. The Nails should cross distal to the fracture site(4-6 $\mathrm{cm}$ distal) Rotate the $\mathrm{T}$ handle or manipulate the limb to direct the Pins into the opposite fragment. If the first is impeded try the second with the aid of an image intensifier. Ensure both nails are in the canal across the fracture site. When they pass the fracture level, release traction, pushing nails farther and fixing their tips in the spongy tissue of the metaphysis, without their passing through the physis. Small distractions can be corrected by rotation of the pins. Avoid residual angulation by ensuring that the nails are introduced at the same level, so that they have identical curvatures. Leave the distal portion of the nails slightly protruding for ease of removal.

If the technique is performed correctly the fracture is finally stabilized by two nails, each with three points of fixation. This fixation is elastic but sufficiently stable to allow automatic small position corrections by limited movements during the limb"s loading.

\section{Results}

Initially 123 patients were included in this study with informed consent. 87 patients were treated with early spica cast (Within one week) and remaining 36 patients were treated with close reduction \& internal fixation with Titanium Elastic nail under $\mathrm{C}$-arm control. twelve patients from spica cast group and 3 patients from operative group lost from follow up were excluded from this study. Remaining 108 patients were followed up properly up to 6 to 18 months after the application of spica or TENS nailing. In spica cast group 74 were male and 13 were female child. Mean age of this group was 7.8 years. In operative group 29 were male and 7 were female child with mean age of 8.2 years .Follow up period in spica cast group was 10 to 18 months and in operative group it was 6 to 13 months. Average time of union in spica cast group was 8 to 14 weeks (mean 10.2 weeks). In operative group average union time was 7 to 10 weeks (mean 8.4 weeks). Mean range of knee joint motion in spica cast group was $137.4^{\circ} \pm 5.4^{\circ}$ and in operative group it was $127.3^{\circ} \pm 9.6^{\circ}$. Two patients developed infection in operative group and none in spica cast group. Infection was superficial and was cured with oral antibiotics. Pressure sore were found in 9 patients $(10 \%)$ of spica cast group at the time of cast removal.In 6 patients $(3.67 \%)$ of spica cast group delayed union was noted where all patients in operative group got union within 3 months time. Fourteen patients $(12.55 \%)$ in spica cast group got malunion whereas in operative group malunion was noticed not even in a single case. $1.5 \mathrm{~cm}$ shortening to $1 \mathrm{~cm}$ lengthening was noted in 10 patients

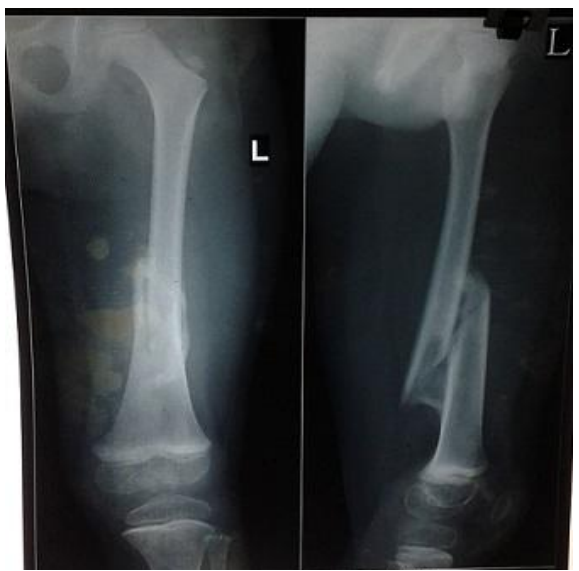

Fig1. Fracture shaft of Femur

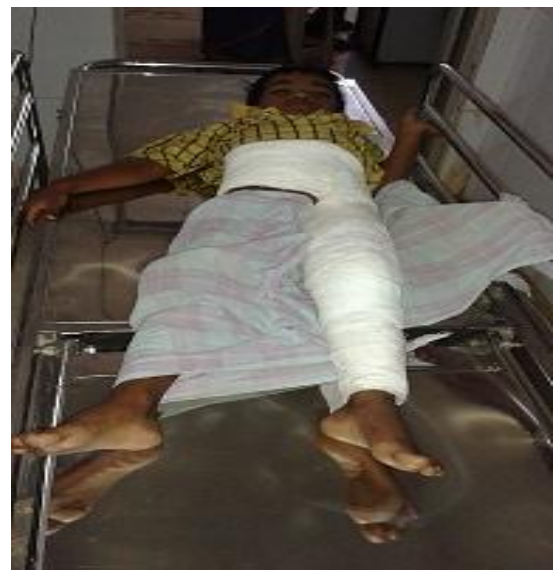

Fig 2. Spica application
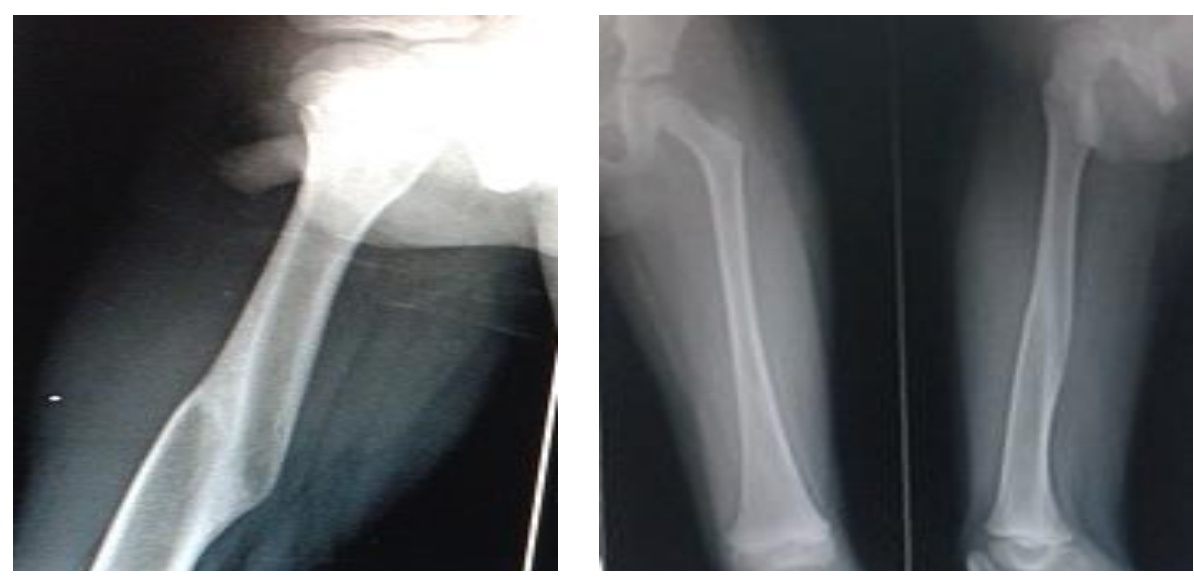


\section{Discussion}

Although spica casting is traditionally used for femoral-shaft fractures in children, recent studies have shown its possible effects on social, economic, educational, and emotional costs. In contrast, elastic intramedullary nailing of femoral-shaft fractures has gained extensive popularity because of its better clinical and psycho-socioeconomic outcomes with lower risk of complications ${ }^{(6-8)}$ In our study, we showed the benefits of the TEN surgical method versus traction and spica casting with respect to time of union, time to start walking, limb length discrepancy, complications. Our findings were in agreement with the results of many studies that showed the efficacy and benefits of elastic nails for treating femoral-shaft fractures.

Ligier et al used elastic intramedullary nail (anterograde or retrograde) with Kirschner wires or pins ${ }^{(9)}$ They reported more desirable outcomes in 120 femoral-shaft fractures treated with TEN. In Reeve et al study, 41 patients with femoral fractures were treated with traction and casting, and 49 cases underwent intra medullary nailing surgery. 9 They showed complications were higher in the traction and casting group in comparison with the group undergoing surgery.

In our study, the duration of hospital stay was longer in operative than the traction and spica cast group. This is in contrary with other studies which reported shorter hospital stays with TEN, but is in conformity with Saseendar's study ${ }^{(10-13)}$ This difference was due to the fact that in our institute plaster room day was earlier than routine ot and long due list of surgery and the spica patients were usually discharged a day or two following spica casting after assessing for the presence of plaster-of Paris related complications.

Our findings showed shorter time of union and weight bearing in the TEN group compared with the spica casting group. It is probably because of better contact of the fracture surfaces and anatomical reduction in patients who underwent TEN surgery. In our study, a higher rate of malunion and limb length discrepancy was observed in the traction and spica group compared with the TEN group. This finding conforms to the results of a similar study conducted by Kirby et al(14) .

Flynn score was better in operative group. Common causes of poor Flynn score in spica cast group were malunion and limb length discrepancy. Our study had certain limitations. Treatment cost, time to return school, parents satisfaction were not measured in either group. As with any other new procedure, we had a small sample size, and thus the results could show falsely high complication rates. Flexible intramedullary nailing offers several advantages including a better reduction, dynamic stabilization, and short hospital stay with early functional recovery, lower treatment costs and a simplified implant removal. The technique is usually closed and minimally invasive. The minimal operative trauma, the undisturbed periosteal and endosteal vasculature and nail elasticity favours rapid fracture union in the paediatric age group. But the uncertain control of rotation, difficulty in fixation of more proximal and distal fractures, maintenance of length in comminuted and unstable fracture pattern, frequent impingement of implant and requirement of another procedure for implant removal are some of the disadvantages of this procedure. It is preferable to use these implants till 12 years of age in length stable mid shaft femoral fractures due to the results of recent studies favouring plate over elastic nails in closed fracture. In a retrospective review of 40 children ( 46 femur fractures), aged 4 to 10 years were treated with open reduction and plate fixation. There were no nonunions. Complication included one case of osteomyelitis and one refracture. They observed limb- length discrepancy averaging $1.2 \mathrm{~cm}$ (range 0.4-1.8 $\mathrm{cm}^{\text {.(15) }}$ A study on 15 femoral shaft fractures with multiple injuries or a head injury, managed with compression plating concluded that plate fixation of the femur is a good treatment option for children who have a femoral shaft fracture associated with major head injury or multiple injuries, or both.26comminuted fractures of shaft and for fractures that are more distal or proximal in location.

This method is based on sound biomechanics. The intramedullary position of the implant places it more in line with the weight bearing forces thereby reducing the tendency of the fracture to settle in a deformed position. The excellent biomechanics is reflected by the absence of implant failure. The risk of infection is negligible in TENS as the incision is far away from the fracture and it is a closed technique.

Patients are more comfortable from $1^{\text {st }}$ post operative day onwards because of least traumatic nature of the procedure. Early ambulation is one of the advantages of the Titanium Elastic nailing. This help to minimise the duration of hospital stay, complications and better rehabilitation than spica casting. Thus TENS is a simple, easy, rapid, reliable, physiological and physeal protective method for management of paediatric femoral fractures with better functional outcome and lesser complication rate as compared to Spica casting.

\section{Biomechanics Of Fracture Healing In Children}

Healing process is faster in paediatric bones compared to adult bones. Diaphyseal Fracture During the initial trauma, bone fractures, some blood vessels are ruptured, and the adjacent soft tissue is torn. Fracture hematoma results from bleeding of bone and soft tissue. According to some authors it is an important determining factor for healing. For others, it does not have an active role this is based on the finding that near normal healing can be achieved in hemophilic patients, and in patients who have undergone open internal fixation. During the early acute inflammatory phase, histamine and other chemical mediators are released, which 
aggravates the initial devitalization of bone and soft tissue. Formation of external or periosteal callus actually initiates the repair process by forming encircling collars of callus at both ends of bone fragments, in the living bone area. These wedge-shaped collars gradually approach each other until they meet, and union is established in a few weeks. Metaphyseal Fractures the metaphysis acts as a damper between the epiphysis and the diaphysis. It is vulnerable to compressive forces (mainly). The fracture surface is generally wider, more bleeding, and has a more transverse orientation in the metaphysis than in the diaphysis. As result, it is more stable after reduction; actually, internal fixation is just this "little something" that protects against secondary displacement.

Hip spica is a orthopaedic cast used to immobilise the Hip or Thigh. It is used to facilitate healing of injured Hip joint or fractured Femur.A Hip spica includes the trunk of the body and one or both legs.

\section{Advantages Of Spica Cast :-}

Low cost ,Excellent safety profile Very high rate of good results ,

Healing time

Flexible Intramedullary Nailing :-The FIN method, also termed Métaizeau technique, Nancy technique , or ESIN technique (mainly in Europe) was introduced in the 1980s

Advantages Of Tens:-The role of an image intensifier is another noteworthy point in titanium nailing.. TENS by closed method, using an image intensifier is ideal, but when it is not available, minimal incision at fracture site makes reduction and nail insertion easy. In open fractures with major wounds, the problem is smaller.

\section{Conclusion}

Treatment for pediatric femoral shaft fractures has changed dramatically over the past several decades. The shift from non-operative treatment with prolonged traction followed by spica casting to immediate spica casting or operative fixation has decreased lengths of hospitalization and the time to return to normal activities. Nonoperative treatment with immediate In modern era of fashion and comfort, titanium elastic nail treatment stands far better than spica cast for treatment of diaphyseal femoral fractures. TEN takes lesser time for union, has minimal limb length discrepancy and malalignment, allows earlier rehabilitation and return to activities of day to day life than spica cast treatment. Patient feels very much comfortable after TEN treatment as compare to spica treatment. Parents of children, treated with TEN, remained much happier than the other group. Convenience of micturition \& defecation in TEN group, also make it better than spica treatment. Thus TEN treatment proved to be superior than spica cast treatment for diaphyseal femoral fractures.

The following conclusions have been arrived at after comparing the overall results of this study with that obtained by other authors.

TENS is less traumatic, gentle and one of the simplest methods known. It is an ideal technique for the treatment of paediatric femoral shaft fractures as it maintains good alignment.

\section{Reference}

[1]. Hinton RY, Lincoln A, Crockett MM, Sponseller P, Smith G (1999) Fractures of the femoral shaft in children. Incidence, mechanical, and sociodemographic risk factors. J Bone Joint Surg Am 81:500-509.

[2]. Flynn JM, Skaggs DL, Sponseller PD et.al. The operative management of pediatric fractures of the lower extremity. J Bone Joint Surg (Am) 2002; 84:2288-2300.

[3]. Rockwood \& Wilkin's Fractures in Children. Femoral shaft fractures.6th Ed.893-936.

[4]. Roop Singh, SC Sharma, Magu NK, Amit Singla. Titanium elastic nailing in pediatric femoral diaphyseal fractures. Ind J Ortho 2006; 40(1): 29-34

[5]. Heinrich SD, Drvaric DM, Darr K, MacEwen GD. The operative stabilization of pediatric diaphyseal femur fractures with flexible intramedullary nail: A proapective analysis. J Pediatr Orthop 1994; 14(4):501-507.

[6]. Flynn JM, Luedtke LM, Ganley TJ, Dawson J, Davidson RS, Dormans JP, et al. Comparison of titanium elastic nails with traction and a spica cast to treat femoral fractures in children. J Bone Joint Surg Am. 2004;:770-7.

[7]. Buechsenschuetz KE, Mehlman CT, Shaw KJ, Crawford AH, Immerman EB. Femoral shaft fractures in children: traction and casting versus elastic stable intramedullary nailing. J Trauma. 2002;53:914-21

[8]. Wright JG. The treatment of femoral shaft fractures in children: a systematic overview and critical appraisal of the literature. Can J Surg. 2000;43:180-9.

[9]. Ligier JN, Metaizeau JP, Prevot J. Closed flexible medullary nailing in pediatric traumatology. Chir Pediatr. 1983;24:383-5

[10]. Reeves RB, Ballard RI, Hughes JL. Internal fixation versus traction and casting of adolescent femoral shaft fractures. J Pediatr Orthop. 1990;:592-5

[11]. Greisberg J, Bliss MJ, Eberson CP, Solga P, d'Amato C. Social and economic benefits of flexible intramedullary nails in he treatment of pediatric femoral shaft fractures. Orthopedics. 2002;25:1067-1073

[12]. Herndon WA, Mahnken RF, Yngve DA, Sullivan JA. Management of femoral shaft fractures in the adolescent. J Pediatr Orthop. 1989; :29-32.

[13]. Saseendar S, Menon J, Patro DK. Treatment of femoral fractures in children: is titanium elastic nailing an improvement over hip spica casting. J Child Orthop. 2010; :245-51.

[14]. Kirby RM, Winquist RA, Hansen ST Jr. Femoral shaft fractures in adolescents: a comparison between traction plus cast treatment and closed intramedullary nailing. J Pediatr Orthop. 1981; 193-7.

[15]. Eren OT, Kucukkaya M, Kockesen C. Open reduction and plate fixation of femoral shaft fractures in children aged 4 to 10. J Pediatr Orthop 2003; 23(2): 190-3. 